\title{
IL RECEPIMENTO DELLA DIRETTIVA RIMPATRI IN ITALIA
}

\author{
Paolo Morozzo della Rocca*
}

Nel giugno 2011 il governo italiano - costretto da un terremoto giurisdizionale il cui epicentro è costituito dalla sentenza della Corte di giustizia sul caso Hassen El Dridi (alias Soufi Karim) contro l'Italia ${ }^{1}$ - decideva molto tardivamente di procedere al recepimento della direttiva 115/2008, modificando obtorto collo il suo precedente avviso, secondo cui la normativa italiana non avrebbe avuto bisogno di alcuna iniziativa di recezione perché già adeguatasi alla direttiva europea in anticipo e per virtù propria.

Si provvedeva quindi al non desiderato recepimento ricorrendo allo strumento del decreto legge (invece di seguire la via più consueta del confronto parlamentare seguito dal decreto legislativo). Un percorso velocissimo di produzione normativa, subito efficace e sicuramente corrispondente al bisogno; cioè alla necessità di uscire dal vuoto normativo creatosi con la rammentata sentenza del giudice dell'Unione europea

Il giudice comunitario aveva infatti dichiarato, in quella sentenza (seguita poi, come è noto, da altre decisioni dello stesso tenore ${ }^{2}$ ), l'incompatibilità con la Direttiva n.115 delle pene detentive all'epoca previste dall'Italia nei riguardi degli immigrati che non avevano ottemperato al provvedimento di espulsione con annesso ordine di allontanamento. Ma le inadempienze dell'Italia erano di certo più complessive, non esistendo all'epoca alcuna disposizione che prevedesse e incoraggiasse I'allontanamento volontario dello straniero irregolarmente soggiornante, realizzando così quella accettabile gradualità degli interventi di contrasto all'immigrazione irregolare che la Direttiva n.115 richiedeva agli Stati membri.

\footnotetext{
* Professore ordinario di Diritto Civile, Università di Urbino/Italia.

${ }^{1}$ Corte di Giustizia dell'Unione Europea, Sez. I, Sentenza del 28 aprile 2011. H.E.D. - Italia

${ }^{2}$ Corte di giustizia dell'Unione europea, sez. III, ord. del 21 marzo 2013; Corte di giustizia dell'Unione europea, Grande sezione, sentenza del 6 dicembre 2011, A.A. - Préfet du Val-de-Marne (Francia).
} 
Come è facile intuire già dalle considerazioni di contesto sin qui svolte, il Decreto legge 23 giugno 2011, n.89 non ha però realizzato un buon recepimento della direttiva 2008/115/CE, la quale è stata presa in considerazione soprattutto nelle sue parti che consentivano di introdurre ulteriori inasprimenti nella disciplina domestica, mentre è stata fortemente sottovalutata nelle sue (pur limitate) aperture ad approcci più modulati.

Uniche note positive, forse, sono quelle costituite dalla novella dell'Art. 5 , co. 6 del testo unico sull'immigrazione che legifica quanto già stabilito a livello regolamentare circa la facoltà del questore di rilasciare un permesso di soggiorno per motivi umanitari, sia nei casi di pericolo nell'eventualità di un rimpatrio sia in altri casi, in nessun modo tipizzabili, nei quali, dalla documentazione presentata dall'interessato, risultino "oggettive e gravi situazioni personali che non consentono l'allontanamento dello straniero dal territorio nazionale".

Tale facoltà va letta sicuramente alla luce dell'Art. 6, co. 4 della Direttiva, ove si dispone che

in qualsiasi momento gli Stati membri possono decidere di rilasciare per motivi caritatevoli, umanitari o di altra natura un permesso di soggiorno autonomo o un'altra autorizzazione che conferisca il diritto di soggiornare a un cittadino di un paese terzo il cui soggiorno nel loro territorio è irregolare. In tali casi non è emessa la decisione di rimpatrio. Qualora sia già stata emessa, la decisione di rimpatrio è revocata o sospesa per il periodo di validità del titolo di soggiorno o di un'altra autorizzazione che conferisca il diritto di soggiornare.

Positivo è, inoltre, l'aver previsto all'Art.10 bis, del testo unico la non punibilità per il reato di presenza irregolare dello straniero identificato come irregolare "durante i controlli della polizia di frontiera, in uscita dal territorio nazionale"; nonché l'aver disposto, all'art.13, che l'espulsione non sia disposta (né tanto meno eseguita, qualora il provvedimento sia stato già adottato) "nei confronti dello straniero identificato in uscita dal territorio nazionale durante i controlli di polizia alle frontiere esterne".

Ad essere tradita è però la finalità, almeno in apparenza strategica, contenuta nella direttiva e mirante a stabilire un sistema ben graduato di misure miranti all'allontanamento dello straniero, all'interno del quale I'allontanamento coattivo dovrebbe costituire misura residuale, facendo così eccezione alla facoltà di rimpatrio volontario. Non v'è dubbio, infatti, che la disciplina italiana ad oggi vigente continui a considerare come ordinarie 
le procedure di espulsione coattiva, relegando a casi davvero eccezionali le ipotesi di rimpatrio volontario.

Ciò accade sia perché i requisiti richiesti per accedere alle misure di allontanamento volontario (disponibilità di un'abitazione, regolarità e validità dei documenti di viaggio, dimostrazione di risorse economiche sufficienti, solo per citare quelli più ordinari) sono così esigenti da non poter essere riempiti che in casi del tutto eccezionali; sia perché i vantaggi riconosciuti allo straniero che allontanandosi spontaneamente evita allo Stato spese ingenti e oneri di fare molto gravosi sono praticamente nulli: egli pure è infatti destinatario di un divieto di reingresso che non potrà, come per gli espulsi, essere inferiore ad un periodo di tre anni e maggiore di cinque anni; divieto di cui - è vero - i rimpatriati volontari potranno chiedere la revoca una volta lasciata I'Italia, ma senza il diritto di ottenerla. Davvero troppo poco come incentivo ad attivarsi per poter essere mandati via!

Mentre le misure previste dalla direttiva riguardo ai rimpatri volontari sono state oggetto di un recepimento avaro e forse infedele, quelle volte a consentire la detenzione amministrativa degli stranieri da espellere sono state invece recepite sino alla soglia temporale massima consentita di 18 mesi, tanto da risultare a tutt'oggi, nonostante la loro provata inefficacia, come il nuovo pilastro delle politiche italiane di contrasto all'immigrazione irregolare assieme alle politiche degli accordi di riammissione con i paesi di provenienza (queste ultime sicuramente più efficaci e comunque necessarie).

Un pilastro ben fragile in ragione delle contraddizioni tra l'enunciazione teorica del minor ricorso possibile al trattenimento ed i meccanismi di esecuzione configurati dal decreto legge.

La direttiva - definita da alcuni "della vergogna" proprio con riferimento a questo esagerato tempo di privazione della libertà personale - connette a questa aspra e prolungata misura detentiva limiti e condizioni di praticabilità che non sembrano essere stati adeguatamente recepiti dal legislatore nazionale che, al contrario, sembra avere perseguito una sua precisa volontà elusiva al riguardo.

Non stupisce, dunque, che l'Amministrazione dell'Interno sia stata già chiamata a risarcire il danno da "ingiustificato trattenimento" per non avere esattamente valutato l'opportunità (rectius: I'utilità) del trattenimento ${ }^{3}$.

Altre condanne all'Italia potrebbero derivare, oltre che dalla ingiustificata lesione del diritto alla libertà personale di una parte degli "ospiti" trattenuti in detenzione amministrativa, anche dalla lesione di altri

${ }^{3}$ Trib. Roma, sent. n. 5764 del 15 marzo 2013 
diritti, primo fra i quali il diritto alla salute, essendo assai misera la qualità dell'ospitalità coatta oggi praticata nei diversi centri di espulsione italiani e ben scarsa la qualità della cura.

Un'ultima annotazione: la direttiva n.115 si avvede del problema rappresentato da coloro che, pur risultando espellibili in diritto non lo sono in fatto, temporaneamente o definitivamente. Un realismo banale, ma non per questo scontato, che risulta comunque coerente all'obiettivo di contrastare l'irregolarità con tutti i mezzi, ricorrendo al rimpatrio quando ciò sia possibile oppure sanando le situazioni altrimenti non risolvibili in altro modo. Di tale approccio è significativo in primo luogo il considerando n. 12 della Direttiva, dedicato agli stranieri "il cui soggiorno è irregolare ma che non è ancora possibile allontanare". Per il cui sostentamento gli Stati membri sono invitati ad occuparsi, affermando infine che "affinché possano dimostrare la loro situazione specifica in caso di verifiche o controlli amministrativi, tali persone dovrebbero essere munite di una conferma scritta della loro situazione".

La preoccupazione contenuta al considerando n. 12 è (solo parzialmente) ribadita dall'Art. 14, par. 2, ove è disposto che in caso di rimpatrio volontario prorogato, così come nel caso di qualsiasi allontanamento differito nel tempo, gli Stati membri confermino per iscritto la proroga del soggiorno o la sospensione dell'allontanamento. Pur se avara nei termini utilizzati, la norma parrebbe disporre il rilascio nelle mani dell'espulso dimostratosi di fatto inespellibile di un documento attestante l'inevitabilità, se non la provvisoria regolarità, del suo soggiorno nel Paese membro dove si trova.

Sebbene oscurata dal tenore minimalista delle parole usate (e degli effetti giuridici connessi), la norma appare sufficientemente concreta e circostanziata da poter pretendere un'efficacia self executing nei singoli Stati. I casi in cui potrebbe esserne pretesa l'applicazione non sono poi così rari. Si pensi, ad esempio, all'allontanamento differito di diversi mesi, per consentire al figlio dello straniero espulso di terminare l'anno formativo in corso, se non addirittura il ciclo di studi.

Ma ancor più frequente - a giudicare dalle statistiche riguardanti la percentuale dei rimpatriati sul totale degli stranieri detenuti sino ad oggi nei c.i.e. ${ }^{4}$ - è il caso dello straniero che, trascorso il periodo massimo di detenzione amministrativa consentita (non necessariamente coincidente,

\footnotetext{
${ }^{4}$ Circa il 50\% circa dei trattenuti non viene espulso ma rilasciato sul territorio nazionale con l'ordine di provvedere da sé al proprio allontanamento.
} 
come si è visto, con la soglia temporale "estrema" dei diciotto mesi) debba essere rilasciato sul territorio nazionale senza alcuna reale prospettiva di allontanamento spontaneo.

Attribuire a questo soggetto, rivelatosi di fatto (almeno nel breve tempo) inespellibile, un documento, pur provvisorio, sia di soggiorno che di identificazione (sia pure non probante la sua effettiva identità, rimasta forse celata) potrebbe essere assai più conveniente, anche solo ai fini della sicurezza pubblica, che lasciarlo circolare senza alcun documento di immediata identificazione.

Più delicata è invece la valutazione riguardo alla mancata trasposizione dell'Art. 6, par. 5 della direttiva, ove è disposto che "qualora un cittadino di un paese terzo il cui soggiorno nel territorio di uno Stato membro è irregolare abbia iniziato una procedura per il rinnovo del permesso di soggiorno o di un'altra autorizzazione che conferisce il diritto di soggiornare, lo Stato membro in questione valuta l'opportunità di astenersi dall'emettere una decisione di rimpatrio fino al completamento della procedura". Si tratta di una disposizione non facoltativa per gli Stati membri ai quali è dunque imposto di operare detta valutazione, con I'onere implicitamente affermato dalla norma di dimostrare che tale valutazione è stata compiuta esplicitandone le ragioni nella motivazione del provvedimento. Anche in questo caso siamo di fronte ad una disposizione self executing, che rende illegittimo il provvedimento di espulsione successivo alla domanda di rinnovo o di rilascio del permesso di soggiorno, ove detto provvedimento non dia conto di tale domanda e delle ragioni per le quali è stato ugualmente adottato. 